
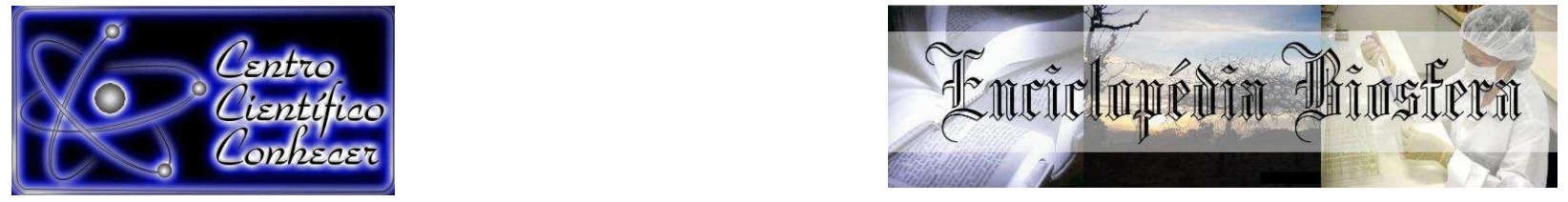

\title{
ESTIMATIVA DA BIOMASSA DE BAMBUS COM CLASSIFICADORES BASEADOS EM INSTÂNCIA E MODELOS DE REGRESSÃO
}

\author{
Matheus Melzer Theodorovicz ${ }^{1}$; Carlos Roberto Sanquetta ${ }^{2}$, Mateus Niroh Inoue \\ Sanquetta ${ }^{1}$; Ana Paula Dalla Corte ${ }^{2}$; Jaime Wojciechowski ${ }^{3}$
}

1 - Acadêmico de Engenharia Florestal da Universidade Federal do Paraná

2 - Eng. Florestal, Dr., Professor da Universidade Federal do Paraná

(carlossanquetta@gmail.com)

3 - Matemático, Dr., Professor da Universidade Federal do Paraná

Av. Lothário Meissner, 900 - Jardim Botânico,Curitiba - PR, 80.210-170 - Brasil

Recebido em: 03/10/2016 - Aprovado em: 21/11/2016 - Publicado em: 05/12/2016 DOI: 10.18677/EnciBio 2016B 047

\begin{abstract}
O objetivo deste trabalho foi testar técnicas Classificadoras Baseadas em Instância, conhecidas como KNN (K Nearest Neighbor) ou Vizinhos Mais Próximos, para a estimação da biomassa de diferentes espécies de bambu e compará-las com diferentes modelos alométricos. Para testar as técnicas de KNN foi utilizado o software JCarbon, disponível na web. A técnica produz estimativas baseadas em valores próximos de plantas conhecidas, cujos vetores formados por variáveis mensuradas nas plantas indicam na média o resultado da predição. Para calcular a distância $(d)$ entre os vetores, utilizou-se as distâncias Euclidiana, Euclidiana Quadrática, Manhattan e Chebychev. Ademais, adicionou-se o número de vizinhos mais próximos, sendo $3,5,7,9$ e 11 , e, ainda ponderações $1 / d$ e $1 / d^{2}$ que objetivam analisar o efeito de transformações nas distâncias. Para o teste foram usados dados individuais de plantas de três espécies de bambu: Bambusa vulgaris, Bambusa oldhamii (Fazenda Canguiri/UFPR) e Phyllostachys aurea (Campus Botânico/UFPR), das quais foram medidos diretamente o diâmetro à altura do peito, a altura total e a biomassa seca aérea total de 30 indivíduos de cada espécie. Combinando distâncias, vizinhos e ponderações, obteve-se 44 combinações de resultados. Destas, se destacaram as simulações com 3 vizinhos, ponderação $1 / d$ para Euclidiana, Euclidiana Quadrática e Chebychev, já para a distância de Manhattan, se destacou a simulação com o mesmo número de vizinhos, porém com ponderação $1 / d^{2}$. Os resultados foram comparados utilizando-se diferentes critérios de seleção, os melhores resultados para a técnica KNN apresentaram resultados satisfatórios, porém, inferiores quando comparados aos modelos alométricos. Portanto, conclui-se que as técnicas KNN geram estimativas semelhantes aos modelos alométricos, mas que no presente caso o modelo de regressão (4) foi superior quanto aos critérios de seleção de modelos $\mathrm{R}^{2}$ corrigido/recalculado, Syx\%, AIC e BIC, além da validação, gerando estimativas mais acuradas.
\end{abstract}

RESUMO

PALAVRAS-CHAVE: inteligência artificial, modelagem, Poaceae. 


\title{
ESTIMATING BIOMASS OF BAMBOOS WITH DISTANCE-BASED CLASSIFIERS AND REGRESSION MODEL
}

\begin{abstract}
The aim of this study was to test Instance-Based Classifiers, known as KNN (K Nearest Neighbor) to estimate the biomass of different bamboo species and compare it with allometrics models. To test the KNN techniques we used the JCarbon software, available on the web. The technique produces estimates based on closest values to a given and known plant, whose vectors formed by variables measured in plants indicate the average result of the prediction. To calculate the distance $(d)$ between vectors, we used the Euclidean distance, Quadratic Euclidean, Manhattan and Chebychev. We also evaluated following number of nearest neighbors: 3, 5, 7, 9 and 11 , with weighting $1 / d$ and $1 / d^{2}$, aimed at examining the effect of changes in distances. For the test were used data from three bamboo species: Bambusa vulgaris, Bambusa oldhamii (Canguiri Farm / UFPR) and Phyllostachys aurea (Campus Botanical / UFPR), which were measured directly the diameter at breast height, total height and dry aboveground biomass of 30 individuals of each species. Combining distances neighbors and weights afforded 44 result combinations. All of them stand out: 3 neighbors and weighting 1/d to Euclidean, Quadratic Euclidean and Chebychev and the weighting $1 / d^{2}$ to Manhattan Distance, with the same number of neighbors. The results were compared using different selection criteria, the best results were obtained with the allometric models. It was concluded that KNN techniques generate estimates similar to the traditional allometric model, but in the present case the allometric model (4) was more accurate, presenting higher $\mathrm{R}^{2}$ corrected/recalculated, lower Syx\%, AIC and BIC.
\end{abstract}

KEYWORDS: modeling, artificial intelligence, Poaceae.

\section{INTRODUÇÃO}

Os bambus estão distribuídos de maneira ampla por grande parte do globo terrestre, abrangendo trópicos, sub-trópicos e zonas temperadas (SCURLOCK et al., 2000). Em muitos locais, chamada de "a planta dos mil usos", esta espécie é utilizada de inúmeras formas na sociedade (GRECO \& COMBERG, 2011). Estima-se que na América Latina existam cerca de 10 milhões de hectares de bambu (MOGNON et al., 2014). Apesar de existir grandes áreas com bambus nativos do Brasil, observa-se também um grande potencial para o cultivo de espécies provenientes de outras regiões (SANQUETTA et al., 2014).

O aumento da concentração de gases do efeito estufa na atmosfera, principalmente do dióxido de carbono $\left(\mathrm{CO}_{2}\right)$ está se tornando um sério problema ambiental para a população mundial. Segundo pesquisa do IPCC (2015) - Painel Intergovernamental sobre Mudanças Climáticas, 24\% das emissões de Gases de Efeito Estufa (GEE) vêm da agricultura, florestas e outros usos da terra (Agriculture, Forestry and Other Land Uses - AFOLU em inglês). A maioria dessas emissões provém da conversão de áreas florestadas para a agricultura, pecuária e outras atividades antrópicas. Nesses processos de conversão, a biomassa existente na vegetação é oxidada pela queima ou decomposição, liberando GEEs para a atmosfera. Assim, estratégias são traçadas para evitar tais emissões. Neste sentido, as atividades florestais surgem como um mecanismo de estocagem de carbono pelo maior acúmulo deste na biomassa florestal (remoção) e evitar as perdas pelo desflorestamento e queimadas (redução), atenuando as concentrações de GEEs na atmosfera e suas implicações. 
Os bambus são plantas que estão entre as que possuem maiores taxas de crescimento vegetal (KUEHL \& YIPING, 2012). Assim, estas se tornam efetivas em contribuir com a diminuição dos efeitos causados pelos GEEs (LOBOVIKOV et al., 2012; INBAR, 2014). Em consequência de seu eficiente sistema de fotossíntese, estas plantas conseguem captar grandes quantidades de carbono que estão na atmosfera e fixar em sua biomassa (THOKCOM \& YADAVA, 2015).

Para haver resultados de quão eficientes são os bambus em armazenar carbono, faz-se necessário quantificar sua biomassa. Em razão dessa necessidade, utilizam-se técnicas de mensuração e inventário florestal para obter estimativas de biomassa de áreas com espécies de bambus.

Diante do cenário exposto e se observando a necessidade da obtenção de resultados mais precisos na estimativa da biomassa, o presente trabalho objetivou testar técnicas de Mineração de Dados conhecidas como "K Nearest Neighbor" ou Vizinhos Mais Próximos, para estimar a biomassa de diferentes espécies de bambu e compará-la com o modelo alométrico de Schumacher \& Hall.

\section{MATERIAL E MÉTODOS}

\section{Área de estudo}

O presente estudo foi desenvolvido com dados coletados em duas diferentes localidades. As informações referentes aos indivíduos do gênero Bambusa foram coletadas em um experimento localizado na Fazenda Experimental do Canguiri, localizada no município de Pinhais, Paraná. A área apresenta as seguintes coordenadas de referência: 2523'30"S e 4907'30"W e 688200/7190200, de acordo com o sistema de projeção UTM (datum SAD-69, UTM Fuso 22S), com altitude variando entre 889 e 950 metros acima do nível do mar e inclinação de 3\% a 20\%. De acordo com Köppen, a área do estudo possui tipo climático subtropical (Cfb). $\mathrm{O}$ mês com temperaturas mais baixas possui média de $18^{\circ} \mathrm{C}$ e 0 mês com temperaturas mais alta a média é de $2^{\circ} \mathrm{C}$ (IAPAR, 2016 ). Os dados de Phyllostachys aurea foram tomados no Campus Jardim Botânico da Universidade Federal do Paraná, o qual encontra-se no município de Curitiba, Paraná. O clima da região segundo a classificação de Köppen é do tipo Cfb (SANQUETTA et al., 2015b).

\section{Trabalho de campo}

Durante o ano de 2015 foram coletados dados de 30 colmos de cada espécie (Bambusa vulgaris, B. oldhamii e Phyllostachys aurea) de maneira a representar a distribuição diamétrica. Os colmos selecionados foram derrubados e tiveram as variáveis de diâmetro à altura do peito ou a $1,30 \mathrm{~m}$ do solo (dap) e altura total $(h)$, medidos diretamente com auxílio de paquímetro e trena, respectivamente. Em seguida, a porção acima do solo do material vegetal das plantas foi separada quanto aos seus componentes, sendo estes: colmo, galhos e folhas, e, em seguida, realizou-se a pesagem das massas frescas em balança digital de precisão de $1 \mathrm{~g}$.

\section{Análises de laboratório}

Amostras com cerca de $500 \mathrm{~g}$ foram coletadas para cada componente das plantas e, em seguida, devidamente identificadas. As mesmas foram encaminhadas para laboratório e submetidas à secagem em estufa com circulação e renovação de ar na temperatura de $65^{\circ} \mathrm{C}$ até atingirem peso constante. Utilizou-se da relação entre biomassa fresca e seca das amostras para converter cada compartimento em massa 
seca e assim somadas as massas dos compartimentos obteve-se a biomassa seca total individual $\left(b t_{i}\right)$ usada neste estudo.

\section{Análises em gabinete}

Os dados foram digitados e compilados em planilha eletrônica. Realizou-se a distribuição em classes diamétricas e, de acordo com sua distribuição, 5 indivíduos representativos por espécie foram retirados dos ajustes para a realização da validação do melhor ajuste. Em seguida, os dados dos 75 indivíduos foram processados no software JCarbon (WOJCIECHOWSKI, 2015), o qual utiliza a técnica de DM com KNN para fazer as estimativas de $b t_{i}$ a partir de dap e $h$. Os dados dos indivíduos das três espécies foram concentrados em apenas uma planilha, gerando assim um somatório de 90 dados.

Para estimar os 75 valores de $b t_{i}$, a técnica KNN usa as instâncias da base de dados real mensurada e busca os valores de $b t_{i}$ mais próximos àquele ponto tendo por base as distâncias dos seus vizinhos calculada a partir de dois vetores $p_{i}$ e $q_{i}$ formados pelos valores de dap e $_{h_{i}}$. O software JCarbon permite testar diferentes distâncias para realizar os cálculos, quais sejam: Euclidiana, Euclidiana Quadrática, Manhattan e Chebychev:

- Distância Euclidiana: $d(p, q)=\sqrt{\sum_{i=1}^{n}\left(p_{i}-q_{i}\right)^{2}}$

- Distância Euclidiana Quadrática: $d(p, q)=\sum_{i=1}^{n}\left(p_{i}-q_{i}\right)^{2}$

- Distância Manhattan: $d(p, q)=\sum_{i=1}^{n}\left|p_{i}-q_{i}\right|$

- Distância Euclidiana: $d(p, q)=\max \left|p_{i}-q_{i}\right|$

Fonte: Wojciechowski (2015)

Além das distâncias, o programa possibilita testar o número de vizinhos mais próximos $(3,5,7,9$ e 11) e ponderações da inversa da distância e seu quadrado.

Para efeitos de comparação dos resultados de estimativa de biomassa com a técnica KNN, realizou-se o ajuste de diferentes modelos de regressão (Tabela 1).

TABELA 1 - Modelos de regressão utilizados para a estimativa da biomassa área total de diferentes espécies de bambu.

\begin{tabular}{llc}
\hline Autor & Modelo & Natureza \\
\hline (1) Schumacher-Hall & $\ln \left(b t_{i}\right)=b_{0}+b_{1} \ln \left(\right.$ dap $\left._{i}\right)+b_{2} \ln \left(h_{i}\right)$ & log. \\
(2) Husch & $\ln \left(b t_{i}\right)=b_{0}+b_{1} \ln \left(\right.$ dap $\left._{i}\right)$ & log. \\
(3) Spurr (var. combinada) & $b t_{i}=b_{0}+b_{1} d_{i}{ }^{2} h_{i}$ & aritm. \\
(4) Kopezky-Gehrhardt & $b t_{i}=b_{0}+b_{1} d_{i}^{2}$ & aritm. \\
\hline
\end{tabular}

Nota: $b t_{i}=$ biomassa aérea total $(\mathrm{kg}) ; d a p_{i}=$ diâmetro à altura do peito do colmo $(\mathrm{cm}) ; h_{i}=$ altura total $(\mathrm{m})$.

A fim de se realizar a comparação entre os ajustes, utilizou-se diferentes critérios de seleção, sendo estes o coeficiente de determinação $\left(R^{2}\right)$ corrigido e/ou recalculado quando necessário, erro padrão da estimativa em percentagem (Syx\%), Critério de Informação de Akaike (AIC) e Critério de Informação de Bayseano (BIC), além da análise gráfica dos resíduos. As equações utilizadas para o cálculo destes 
critérios pode ser consultada em Sanquetta et al. (2015a). Os modelos de natureza logarítmica tiveram suas estatísticas recalculadas a partir do Fator de Correção de Meyer, este indispensável para a comparação com modelos de natureza aritmética.

\section{RESULTADOS E DISCUSSÕES}

\section{Relação entre as variáveis}

A análise descritiva das variáveis biométricas das três espécies de bambus estudadas denota que as duas espécies do gênero Bambusa possuem dimensões que se aproximam em termos de diâmetro à altura do peito (dap), altura ( $h t)$ e biomassa $(b t)$. Já a espécie $P$. aurea apresenta menor porte, com menores dimensões no que tange às variáveis mensuradas (Tabela 2). De fato, o bambudourado $(P$. aurea) é tipicamente de menor porte em comparação com as duas outras espécies analisadas (vide também MONASTIER et al., 2015).

TABELA 2 - Estatísticas descritivas das variáveis à altura do peito (dap), altura (h) e biomassa seca individual total acima do solo $(b t)$ para três espécies de bambus (Bambusa vulgaris, B. oldhamii e Phyllostachys aurea)

\begin{tabular}{cccccc}
\hline Espécie & Variável & Média & Mínimo & Máximo & Desvio Padrão \\
\hline \multirow{3}{*}{ B. oldhamii } & dap $(\mathrm{cm})$ & 3,71 & 1,10 & 7,20 & 1,43 \\
& $h(\mathrm{~m})$ & 6,61 & 2,80 & 13,50 & 2,35 \\
& $b t(\mathrm{~kg})$ & 3,60 & 0,43 & 10,85 & 2,54 \\
\hline \multirow{3}{*}{ B. vulgaris } & dap $(\mathrm{cm})$ & 4,23 & 1,90 & 6,80 & 1,45 \\
& $h(\mathrm{~m})$ & 7,80 & 4,00 & 11,20 & 2,33 \\
& $b t(\mathrm{~kg})$ & 4,12 & 0,74 & 9,30 & 2,36 \\
\hline \multirow{3}{*}{ P. aurea } & dap $(\mathrm{cm})$ & 2,78 & 1,40 & 4,60 & 0,80 \\
& $h(\mathrm{~m})$ & 6,91 & 5,10 & 9,18 & 1,02 \\
& $b t(\mathrm{~kg})$ & 1,51 & 0,46 & 3,27 & 0,78 \\
\hline
\end{tabular}

Observou-se uma correlação entre as variáveis dap e $h$ da ordem de $p=0,870$, o que significa dizer que essas duas variáveis apresentam associação significativa ao nível de significância de 0,05 . A correlação entre dap e bt foi muito forte, com coeficiente de correlação linear de 0,951 e forte com $h$, ou seja, 0,829, ambas significativas a 0,05 . Isso denota que a biomassa acima do solo dessas três espécies pode ser explicada pelas variáveis biométricas diâmetro e altura dos seus colmos (Figura 1). Portanto, é factível desenvolver modelos que possam estimar a biomassa (só possível de ser obtida por método destrutivo), a partir de variáveis tomadas diretamente na planta, sem necessidade de técnica destrutiva.

Como se observa pela Figura 1, em termos de diâmetro (dap) e biomassa $(b t)$, as relações dimensionais são semelhantes entre as três espécies. O mesmo não acontece com a altura $(h)$, que apresenta comportamento distinto entre as diferentes espécies.

SANQUETTA et al. (2014) analisaram as correlações entre dap, altura e biomassa aérea de Bambusa oldhamii e $B$. vulgaris. Os autores verificaram coeficientes de correlação do dap com a biomassa da ordem de $p=0,921$ e $p=0,916$ para as duas espécies, respectivamente. MONASTIER et al. (2015), trabalhando 
com $P$. aurea, encontrou coeficiente de correlação de Pearson da ordem de $p=0,80$ entre dap e a biomassa e de $p=0,58$ entre $h$ e a biomassa aérea total individual.

MOGNON et al. (2014), em trabalho análogo com o gênero Guadua, encontraram que a biomassa aérea total individual correlacionou-se fortemente com o diâmetro $(0,99)$ e com a altura das plantas $(0,94)$. Uma vez que este estudo reuniu dados de três espécies, observando-se classes diamétricas diferentes, assim como alturas, pode-se considerar que os resultados das correlações variam caso a caso, mas que diâmetro e altura dos colmos são variáveis biométricas que efetivamente ditam a biomassa aérea dos bambus.
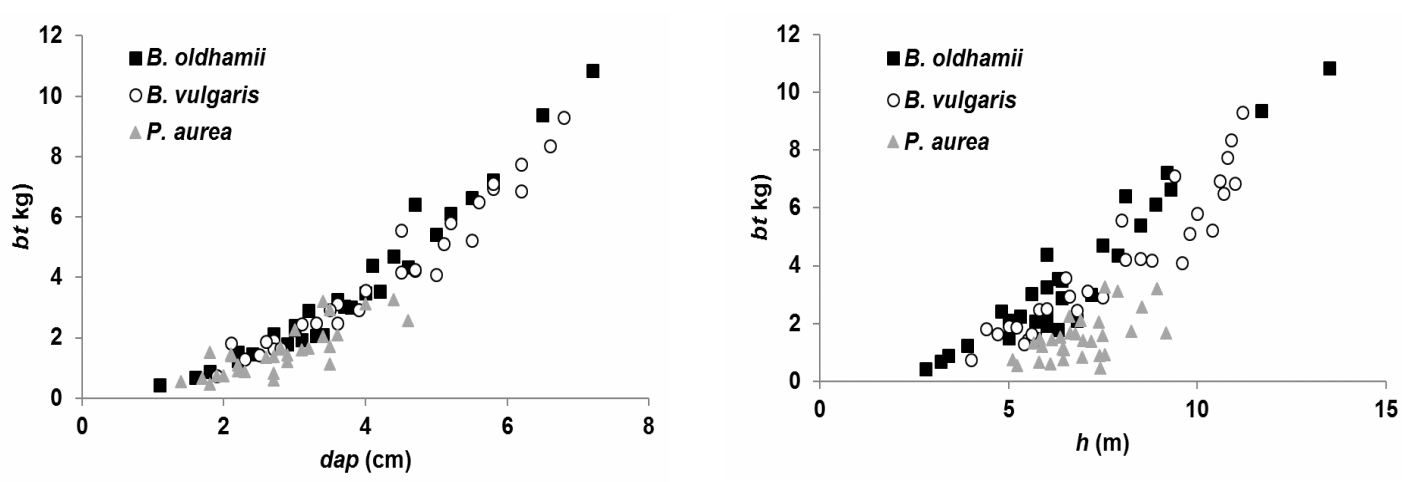

FIGURA 1. Relação do dap (a) e da altura $h$ (b) com a biomassa de três espécies de bambus (Bambusa vulgaris, $B$. oldhamii e Phyllostachys aurea)

\section{Modelagem da biomassa com KNN}

Tendo em vista a explicitada relação do dap e de $h$ com a biomassa, buscouse testar tal relação mediante o emprego da técnica $\mathrm{KNN}$, que vem sendo empregada para esse fim (SANQUETTA et al., 2015b).

Considerando-se as 44 combinações entre distâncias, vizinhos e ponderações de distância, foram gerados resultados gráficos que expressam o comportamento do erro padrão da estimativa (Figura 2). Esses erros variaram de 24,68 a 30,15\% e os menores valores para cada distância foram obtidos através da utilização de três vizinhos, ponderação $1 / d$ para as Distâncias Euclidiana, Euclidiana Quadrática e Chebychev e ponderação $1 / d^{2}$ para a distância Manhattan. Entre estas, a Distância de Chebychev apresentou o menor valor (24,68\%). Enquanto ao coeficiente de determinação $\left(\mathrm{R}^{2}\right)$, os maiores valores foram observados para os resultados sem ponderação e com um vizinho, porém, de maneira geral, seus erros foram os mais elevados. Os resultados para $\mathrm{R}^{2}$ variaram para estas combinações nas magnitudes de 0,9070 a 0,9333, com o maior valor sendo para a Distância Chebychev e a menor para Manhattan. Quanto às combinações que apresentaram os menores erros, os valores para $\mathrm{R}^{2}$ foram inferiores, porém satisfatórios, variando de 0,8315 a 0,8572 .

Verifica-se pelo teste da técnica KNN para estimação da biomassa dos bambus que o emprego de apenas de um vizinho mais próximo resulta em maior erro e maior coeficiente de determinação. O aumento do número de vizinhos para três diminui o erro. Na maioria dos casos a faixa ideal em termo de número de vizinhos fica entre 3 e 5. Nessa faixa a ponderação pelo inverso da distância se comporta melhor, o que não se verifica nas demais. 
MOGNON et al. (2014), em estudo sobre aplicação de KNN para estimar a biomassa de bambus do gênero Guadua, utilizando 38 dados de colmos de duas espécies, encontraram valores semelhantes para Syx \% aos deste trabalho $(23,8 \%)$, da mesma forma para o coeficiente de determinação $(0,81)$
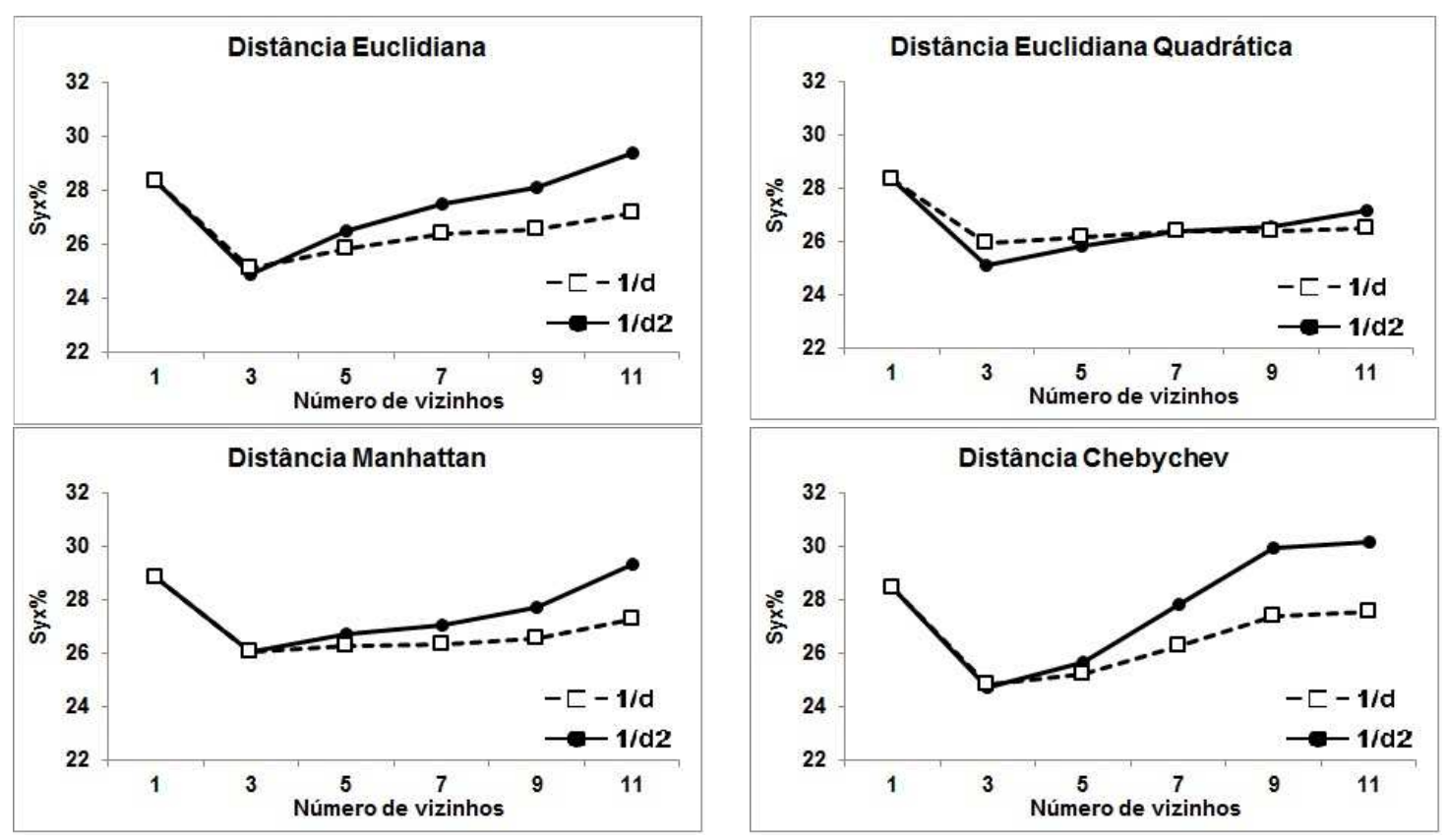

FIGURA 2. Comportamento do erro padrão da estimativa na estimação de biomassa de três espécies de bambus (Bambusa vulgaris, $B$. oldhamii e Phyllostachys aurea) utilizando diferentes modelos de KNN com: (a) Distância Euclidiana; (b) Distância Euclidiana Quadrática; (c) Distância Manhattan; (d) Distância Chebychev

\section{Modelagem da biomassa com modelo de regressão}

Os modelos de regressão linear são muito empregados para estimar não somente o volume de madeira, mas também a biomassa de espécies lenhosas. Neste sentido foram testados quatro modelos de regressão linear para efeito de comparação com os resultados obtidos através da técnica explicitada anteriormente. Após o teste dos modelos, foram geradas as seguintes equações (Tabela 3).

TABELA 3 - Equações ajustadas para a estimativa da biomassa área total de diferentes espécies de bambu.

\begin{tabular}{cccccccc}
\hline Eq. & $\mathrm{b}_{0}$ & $\mathrm{~b}_{1}$ & $\mathrm{~b}_{2}$ & $\mathrm{R}_{\text {corrig/recalc. }}^{2}$ & $\mathrm{~S}_{\mathrm{yx}}(\%)$ & $\mathrm{AIC}$ & $\mathrm{BIC}$ \\
\hline$(1)$ & $-0,9667$ & 1,9425 & $-0,2592$ & 0,7955 & 19,99 & $-68,10$ & 262,50 \\
$(2)$ & $-1,2701$ & 1,7821 & - & 0,8364 & 19,40 & $-72,56$ & 258,04 \\
$(3)$ & 1,0802 & 0,0162 & - & 0,9007 & 23,91 & $-41,21$ & 289,39 \\
$(4)$ & 0,1766 & 0,1996 & - & 0,9406 & 18,50 & $-79,72$ & 250,88 \\
\hline
\end{tabular}

Os ajustes resultaram, de maneira geral, em resultados satisfatórios. Percebe-se que para os modelos 3 e 4 os resultados para $R^{2}$ foram superiores aos ENCICLOPÉDIA BIOSFERA, Centro Científico Conhecer - Goiânia, v.13 n.24; p.525 
demais. Quanto ao Syx\%, o modelo 3 apresentou o menor valor, no caso o mais satisfatório (18,50\%). Os demais modelos variaram entre $19,40 \%$ e $23,91 \%$, sendo estes também mais satisfatórios em comparação com a técnica KNN utilizada, gerando resultados mais acurados. A Figura 5 mostra a distribuição gráfica dos resíduos para a estimação com o melhor modelo de regressão linear e a melhor opção de modelagem com uso da técnica KNN. Observa-se que a distribuição dos resíduos para a regressão linear foi mais equilibrada ao longo da linha de estimação

Diante dos resultados aqui apresentados pode-se afirmar que é preferível a utilização da técnica de regressão linear para estimar a biomassa aérea das espécies Bambusa vulgaris, B. oldhamii e Phyllostachys aurea, pois os erros de estimativa são menores nesse caso e não há presença de vieses. Resultados semelhantes foram obtidos por MOGNON et al. (2014) e, estudo correlato com duas espécies de bambus do gênero Guadua.

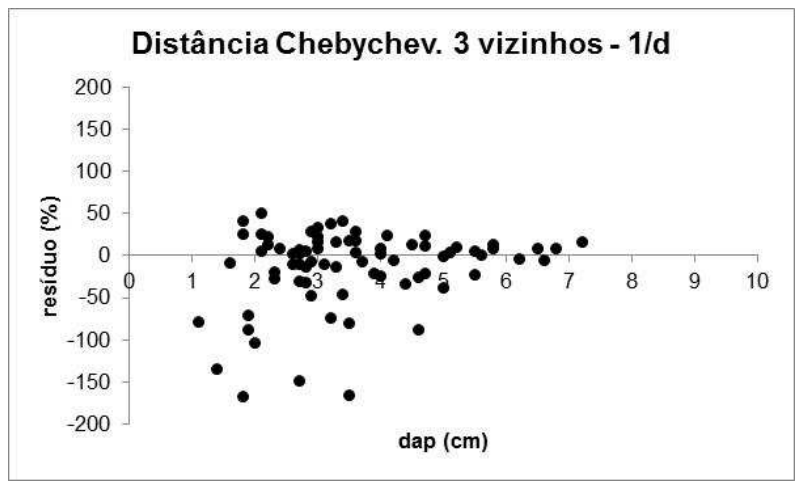

(a)

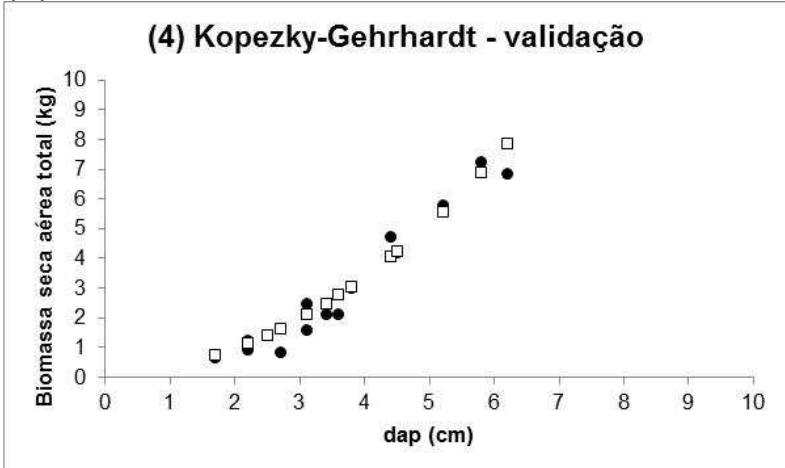

(c)

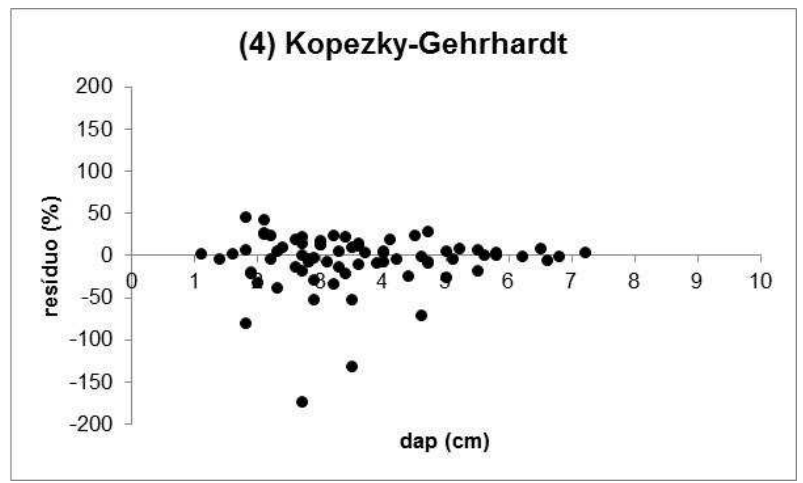

(b)

(4) Kopezky-Gehrhardt - validação

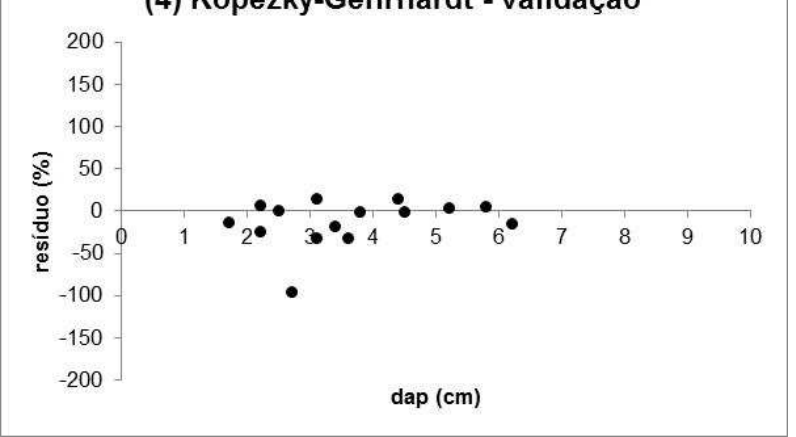

(d)

FIGURA 3. Distribuição gráfica dos resíduos da melhor opção de modelo de KNN (a) e de regressão linear (b). Validação do melhor ajuste (4) para estimativa de biomassa de três espécies de bambus (Bambusa vulgaris, $B$. oldhamii e Phyllostachys aurea) (c e d).

\section{CONCLUSÕES}

Observa-se correlação forte entre a biomassa total e as variáveis biométricas: diâmetro à altura do peito e altura total para o conjunto de dados das três espécies de bambu estudadas (Bambusa vulgaris, B. oldhamii e Phyllostachys aurea), o que viabiliza a estimação por modelagem.

Entre as variantes de KNN, a melhor opção foi aquela que considera 3 vizinhos mais próximos, distância Chebychev e ponderação pelo inverso da distância. 
O modelo de regressão de (4) Kopezky-Gehrhardt testado gerou equação ajustada por regressão linear que superou em performance os modelos de KNN, alcançando menor erro padrão da estimativa para o conjunto de dados considerado nas análises.

\section{REFERÊNCIAS}

GRECO, T. M.; CROMBERG, M. Bambu cultivo e manejo. 1. Ed. Florianópolis. SC: Insular, 2011. $184 \mathrm{p}$.

INSTITUTO AGRONÔMICO DO PARANÁ (IAPAR). Classificação Climática. 2011. Disponível em: $<$ http://www.iapar.br/modules/conteudo/conteudo.php?conteudo=863>. Acesso em: 02/09/2016.

INTERNATIONAL NETWORK FOR BAMBOO AND RATTAN (INBAR). Bamboo: A strategic resource for countries to reduce the effects of climate change. Beijing. 2014. 20p.

INTERGOVERNMENTAL PANEL ON CLIMATE CHANGE (IPCC). Climate Change 2014 - Synthesis Report. IPCC: Genebra. 151p., 2015.

KUEHL, Y.; YIPING, L. Carbon offsetting with bamboo. INBAR Working Paper 71, 25p, 2015.

LOBOVIKOV, M.; SCHOENE, D.; YPING L. Bamboo in climate change and rural livelihoods. Mitigation and Adaptation Strategies to Global Change v.17, p. 261276, 2012. Disponível em: <http://dx.doi.org/10.1007/s11027-011-9324-8>. doi: 10.1007/s11027-011-9324-8.

MOGNON, F; DALLA CORTE, A.P.; SANQUETTA, C.R.; BARRETO, T.G; WOJCIECHOWSKI. J. Estimativas de biomassa para plantas de bambu do gênero Guadua. Revista Ceres, Viçosa, v.61, n.6, p.900-906, 2014. Disponível em: <http://dx.doi.org/10.1590/0034-737X201461060003>. doi: 10.1590/0034737X201461060003.

MONASTIER, S.H.; DALLA CORTE, A.P.; SANQUETTA, C.R.; SANQUETTA, M.N.I.; RUZA, M.S. Modelagem da biomassa individual de Phyllostachys aurea Carr. Ex A.\& C. Rivi're. Enciclopédia Biosfera, Centro Científico Conhecer - Goiânia, v.11, n.21; p. 1290-1297. 2015.

SANQUetTA, C. R.; SANQUeTTA, M. N. I.; DALLA CORTE, A. P.; PÉllico NETTO, S.; MOGNON, F.; WOJCIECHOWSKI, J.; RODRIGUES, A. L. Modeling the apparent volume of bamboo culms from Brazilian plantation. African Journal of Agricultural Research, v.10, n.42, p.3977-3986, 2015. Disponível em: <http://dx.doi.org/10.5897/AJAR2014.9176>. doi: 10.5897/AJAR2014.9176.

SANQUETTA, C.R.; WOJCIECHOWSKI, J.; DALLA CORTE, A. P.; BEHLING, A.; PÉLLICO-NETTO, S.; RODRIGUES. A. L.; SANQUETTA, M N. I. Comparison of data mining and allometric model in estimation of tree biomass. BMC 
Bioinformatics, v.16, n. 247, p.1-9, 2015. Disponível em: <http://dx.doi.org/10.1186/s12859-015-0662-5>. doi: 10.1186/s12859-015-0662-5.

SANQUETTA, M.N.I.; SANQUETTA, C.R.; MOGNON, F.; DALLA CORTE, A.P.; MONASTIER, S.H. Equações hipsométricas para obtenção de comprimento de colmo para Phyllostachys aurea Carr. Ex A.\& C. Rivi're. Enciclopédia Biosfera, Centro Científico Conhecer - Goiânia, v.10, n.19; p. 990-997. 2014.

SCURLOCK, J. M. O.; DAYTONB, D. C.; HAMESB, B. Bamboo: an overlooked biomass resource. Biomass and Bioenergy, n. 19, p. 229-244, 2000. Disponível em: <http://dx.doi.org/10.1016/S0961-9534(00)00038-6>. doi: 10.1186/s12859-0150662-5.

THOKCOM, A.; YADAVA, P. S. Bamboo and its role in climate change. Current Science, v. 108, n. 5, p. 762-763, 2015.

WOJCIECHOWSKI, J. JCarbon-software na web com Data Mining para estimativas de volume, biomassa e carbono em florestas. 2015. Disponível em: $<$ http://biblioteca.versila.com/27762485/jcarbon-software-na-web-com-data-miningpara-estimativas-de-volume-biomassa-e-carbono-em-florestas>. 